\title{
Desempenho dos Programas de Controle da Tuberculose: Revisão Integrativa da Literatura
}

\author{
Melisane Regina Lima Ferreira, ${ }^{1}$ Rafaele Oliveira Bonfim, ${ }^{2}$ Nathalia Halax Orfão ${ }^{3}$
}

\begin{abstract}
RESUMO
O objetivo deste estudo foi identificar e descrever a produção científica acerca do desempenho dos Programas de Controle da Tuberculose (PCTs) em diferentes cenários. Trata-se de uma revisão integrativa da literatura, realizada no mês de fevereiro de 2020, nas bases de dados Lilacs, Pubmed, Cinahl, Scopus e Embase. Foram selecionados estudos primários que abordassem o desempenho do PCT no cenário nacional e internacional, publicados entre 2014 e 2019 nos idiomas português, inglês e espanhol. Foram encontrados 1.267 estudos nas bases de dados, dos quais 23 foram incluídos no estudo. Os artigos encontrados abordavam os seguintes temas: os efeitos da introdução do PCT nos diferentes cenários; avaliação do PCT por meio dos indicadores operacionais e de desempenho; avaliação da satisfação dos usuários, comunidade e profissionais de saúde; análise das estratégias incorporadas aos programas e fatores que afetam seu desempenho. No cenário nacional, identificou-se ainda algumas limitações a serem transpostas: realização do Tratamento Diretamente Observado, alocação de recursos para o PCT prisional, investimento nas equipes da Atenção Primária à Saúde, diagnóstico, assistência clínica e laboratorial. No cenário internacional detectou-se dificuldades estruturais e de processo, como o acesso aos serviços de saúde, busca ativa de casos e manejo da coinfecção TB/HIV, o que refletiu nos resultados obtidos pelos programas. Identificou-se a importância da realização de debates sobre os desafios que permeiam o controle da tuberculose, levando em conta a necessidade de adoção de ações integradas e intersetoriais na efetivação do combate e controle da doença.
\end{abstract}

Palavras-chave: Tuberculose. Avaliação de programas e projetos de saúde. Indicadores de qualidade em assistência à saúde.

\section{PERFORMANCE OF TUBERCULOSIS CONTROL PROGRAMS: INTEGRATIVE LITERATURE REVIEW}

\begin{abstract}
This study aimed to identify and describe the scientific production about the performance of Tuberculosis Control Programs (PCT) in different scenarios. It is an integrative literature review, carried out in February 2020, in the Lilacs, Pubmed, Cinahl, Scopus, and Embase databases. Primary studies were selected that addressed the performance of PCT in the national and international scenario, published between 2014 and 2019 in Portuguese, English, and Spanish. It was possible to find 1,267 studies in the databases, of which 23 were included in the study. The articles found addressed the following topics: the effects of the PCT implementation in different scenarios; PCT assessment using operational and performance indicators; evaluation of the satisfaction of users, community and health professionals; analysis of the strategies incorporated into the programs and factors that affect their performance. In the national scenario, there were also some limitations to be transposed to the realization of the Directly Observed Treatment, allocation of resources to the prison PCT, investment in the Primary Health Care teams, diagnosis, clinical and laboratory assistance. In the international scenario, structural and process difficulties were pointed out, such as access to health services, active search for cases and management of TB/HIV co-infection, which reflected in the results obtained by the programs. The importance of building debates on the challenges that permeate tuberculosis control was identified, taking into account the need to implement integrated and intersectoral actions to combat and control the disease effectively.
\end{abstract}

Keywords: Tuberculosis. Program evaluation. Quality indicators, health care.

\footnotetext{
Autora correspondente. Universidade Federal de Rondônia (Unir). BR 364, Km 9,5. CEP 76801-059 - Porto Velho/RO, Brasil. http://lattes.cnpq.br/ 7787086508381811. https://orcid.org/0000-0003-1694-5124. melisane1206@gmail.com

${ }^{2}$ Escola de Enfermagem de Ribeirão Preto da Universidade de São Paulo (EERP/USP). Ribeirão Preto/SP, Brasil.

3 Universidade Federal de Rondônia (Unir). Porto Velho/RO, Brasil.
} 


\section{INTRODUÇÃO}

A tuberculose (TB) é considerada um grave e persistente problema de saúde pública que se destaca por sua magnitude quando comparada às demais doenças infectocontagiosas. Estima-se que, no ano de 2018, 10 milhões de pessoas tenham adoecido e 1,451 milhão tenham chegado a óbito pela doença, caracterizando-a como a primeira entre as principais causas de morte por agentes infecciosos, superando o HIV/Aids. No Brasil, no mesmo ano, estima-se que 95 mil novos casos da doença tenham ocorrido entre a população, com um total de 4,8 mil óbitos pela TB (WHO, 2019).

Diante da reemergência da TB no cenário nacional, foi oficializado, em 1999, o Programa Nacional de Controle da Tuberculose (PNCT), com os objetivos de reduzir a morbimortalidade e transmissão da doença. Para isso, diversas estratégias foram desenvolvidas a fim de atingir tais objetivos, a exemplo de ações educativas, capacitações, aperfeiçoamento da vigilância epidemiológica, melhorias no Sistema de Informação de Agravos de Notificação (Sinan), mobilização dos gestores, bem como intensificação de ações preventivas em âmbitos federal, estadual e municipal (BRASIL, 2018a).

Além disso, as ações de intervenção voltadas à problemática da TB no Brasil organizam-se por meio dos Programas de Controle da Tuberculose (PCTs), representados nas três esferas, os quais devem estar vigilantes em relação a uma série de indicadores epidemiológicos e operacionais recomendados pelo Ministério da Saúde (MS). Isto possibilita o monitoramento das ações e dos resultados obtidos, tais como o coeficiente de incidência, de mortalidade, cura e abandono, controle de contatos, coinfecção TB/HIV, Tratamento Diretamente Observado (TDO), exames diagnósticos e de acompanhamento, bem como casos de retratamento (BRASIL, 2018b).

Diante dos entraves sofridos na consecução plena dos princípios e diretrizes do Sistema Único de Saúde (SUS), contudo, nota-se a crescente demanda por evidências capazes de contribuir no aprimoramento da prestação de assistência que respondam às necessidades de saúde da população (PAIM, 2013). Nesse sentido, questionar o desempenho de intervenções definidas por meio de programas e políticas de saúde pública é um ato contínuo e instrumento necessário para a concretização de um projeto de saúde integral que contemple indivíduos e comunidades (VIACAVA et al., 2012), visando a assegurar condições adequadas para o desenvolvimento das ações do PCT para o controle da doença.

Este desempenho, em síntese, encontra-se definido com diferentes recortes que perpassam as ações do programa, atributos dos serviços de saúde, atividades voltadas para populações específicas, debates com o enfoque na gestão de serviços e uso de indicadores epidemiológicos e operacionais na saúde pública (ARAKAWA et al., 2015), que diferem de acordo com a complexidade operacional e contexto de instalação do PCT no país.

Assim, considerando a importância da construção de debates sobre os desafios que ainda permeiam o sistema de saúde e a sociedade, visando à criação de ações integradas e intersetoriais na efetivação do combate e controle da doença, o objetivo deste estudo foi identificar e descrever a produção científica acerca do desempenho do PCT em diferentes cenários.

\section{MÉTODO}

Trata-se de uma revisão integrativa da literatura, utilizada em razão do seu potencial para organizar, sintetizar e construir novos conhecimentos, a partir da produção de um saber sistematizado e fundamentado, com o objetivo de identificar lacunas do conhecimento e explorar métodos de pesquisas utilizadas com sucesso (PAULA; PADOIN; GALVÃO, 2016, p. 52), a fim de contribuir na análise crítica do objeto proposto.

Nesse sentido, foram adaptadas as recomendações da diretriz Preferred Reporting Items for Systematic Reviews and Meta-Analyses (Prisma) (MOHER et al., 2009) para o desenvolvimento desta revisão integrativa, considerando os seis passos, a saber: 1 ) formulação da questão norteadora; 2 ) busca na literatura; 3) extração dos dados dos estudos selecionados; 4) análise crítica; 5) interpretação e síntese dos resultados da revisão; 6) apresentação da revisão integrativa (HOPIA; LATVALA; LIIMATAINEN, 2016).

Para a definição da questão norteadora utilizou-se a proposta da estratégia Pico, em que $\mathrm{P}$ (problema) correspondeu à TB, I (intervenção) ao PCT, C (controle) sem aplicação neste estudo, e $\mathrm{O}$ (outcome ou desfecho) se referiu ao desempenho dos programas. Sendo assim, formulou-se a seguinte questão norteadora: "O que as produções científicas disponíveis na literatura têm abordado acerca do desempenho do PCT nos cenários nacional e internacional?". 
A busca nas bases de dados selecionadas ocorreu no mês de fevereiro de 2020 e foi realizada por duas pesquisadoras independentes, havendo a possibilidade de uma terceira pesquisadora, caso houvesse discordância na seleção dos artigos. As expressões de busca foram elaboradas utilizando operadores booleanos AND e OR para a combinação dos descritores indexados no Descritores em Ciências da Saúde (DeCS), Medical Subject Headings (Mesh) e Emtree (Embase Subject Headings) - "Tuberculose" e "Avaliação de Programas e Projetos de Saúde", com seus respectivos sinônimos em inglês e espanhol, e palavras-chave encontradas em buscas prévias nas bases de dados (Quadro 1).

Quadro 1 - Expressões de busca nos idiomas português, inglês e espanhol, utilizadas no processo de busca e seleção dos artigos desta revisão integrativa, 2020

\begin{tabular}{|c|c|}
\hline Idic & Expressões de busca* \\
\hline Português & $\begin{array}{l}\text { Tuberculose OR “Pneumologia Sanitária” OR } \\
\text { "Infecção por Mycobacterium tuberculosis" OR TB } \\
\text { "Avaliação de Programas e Projetos de Saúde" } \\
\text { OR “Avaliação de Programa" OR “Avaliação de } \\
\text { Programas" OR "Avaliação de Projetos" }\end{array}$ \\
\hline Ing & $\begin{array}{l}\text { Tuberculosis OR "Infections, Mycobacterium } \\
\text { tuberculosis" OR "Koch Disease" OR "Kochs Disease" } \\
\text { OR "Mycobacterium tuberculosis Infection" OR } \\
\text { "Mycobacterium tuberculosis Infections" OR } \\
\text { Tuberculoses } \\
\text { "Program Evaluation" OR "Appropriateness, } \\
\text { Program" OR "Effectiveness, Program" OR } \\
\text { "Evaluation, Program" OR “Evaluations, Program" OR } \\
\text { "Family Planning Program Evaluation" OR "Program } \\
\text { Appropriateness" OR "Program Effectiveness" OR } \\
\text { "Program Evaluations" OR "Program Sustainabilities" } \\
\text { OR "Program Sustainability" OR "Sustainabilities, } \\
\text { Program" OR "Sustainability, Program" }\end{array}$ \\
\hline Espanhol & $\begin{array}{l}\text { Tuberculosis OR "Infección por Mycobacterium } \\
\text { tuberculosis" OR TB } \\
\text { "Evaluación de Programas y Proyectos de Salud" } \\
\text { OR "Evaluación de Programas" OR "Evaluación de } \\
\text { Proyectos" }\end{array}$ \\
\hline
\end{tabular}

* As duas expressões nos três idiomas listados foram conectadas por meio do booleano AND.

Fonte: Elaborada pelas autoras (2020).

O levantamento bibliográfico foi realizado nas bases de dados da Literatura Latino-Americana e do Caribe em Ciências da Saúde (Lilacs), Literatura Internacional em Ciências da Saúde (Pubmed), Cumulative Index to Nursing and Allied Health Literature (Cinahl), Scopus e Excerpta Medica Database (Embase), por meio do Portal de Periódicos da Capes, utilizando o acesso remoto via Comunidade Acadêmica Federada (CAFe). Os resultados encontrados foram exportados para o apli- cativo de revisão sistemática on-line Rayyan QCRI da Qatar Computing Research Institute (OUZZANI et al., 2016), para a seleção e análise crítica dos estudos.

Para a seleção dos artigos optou-se pela inclusão de estudos primários disponíveis na íntegra, em livre acesso, publicados no período entre 2014 e 2019, nos idiomas português, inglês e espanhol. Foram excluídos os artigos duplicados, artigos de revisão, de reflexão/debates, monografias, dissertações, teses, comentários, editoriais, cartas, relatórios e manuais.

Posteriormente procedeu-se à leitura dos títulos e resumos a fim de identificar o critério de elegibilidade das publicações: estudos que abordassem aspectos relacionados ao desempenho do PCT nos diferentes cenários nacionais e/ou internacionais. Os estudos considerados elegíveis foram lidos na íntegra para serem incluídos, ou não, na revisão.

A interpretação e síntese dos resultados foi submetida a uma avaliação qualitativa utilizando o instrumento baseado em Ursi (2005), que contempla a identificação do artigo, características metodológicas do estudo, bem como a avaliação do rigor metodológico e principais resultados.

\section{RESULTADOS}

Foram encontrados 1.267 artigos nas bases de dados, dos quais em um primeiro momento foram excluídos 759 estudos por estarem indisponíveis, 262 por estarem inferiores ao período previamente selecionado, 56 por estarem duplicados, 15 por serem artigos de revisão, 5 por serem de reflexão/debate, 4 de cada por serem cartas e editoriais, 2 de cada por serem tese, comentário e manual, 2 por estarem em outros idiomas e 1 dissertação e 1 relatório (Figura 1).

Após a leitura dos títulos e resumos, 125 artigos foram excluídos por não responderem à questão norteadora do estudo. Assim, foram selecionados 27 artigos elegíveis para serem lidos na íntegra, o que possibilitou a inclusão de 23 artigos para serem analisados a fim de subsidiar a revisão de literatura (Figura 1).

Os artigos incluídos nesta revisão foram publicados, em sua maioria, no idioma inglês $(86,9 \%)$, no ano de 2017 (26,1\%), em periódicos internacionais $(69,6 \%)$, dos quais se destacam PLoS ONE, BMC Public Health, International Journal of Mycobacteriology e Global Health Action. Além disso, os estudos foram realizados majoritariamente no cenário internacional, especialmente em alguns dos 30 países que concentram uma alta carga para TB, responsáveis por $87 \%$ de 
Figura 1 - Fluxograma das etapas para a seleção dos artigos desta revisão integrativa, 2020

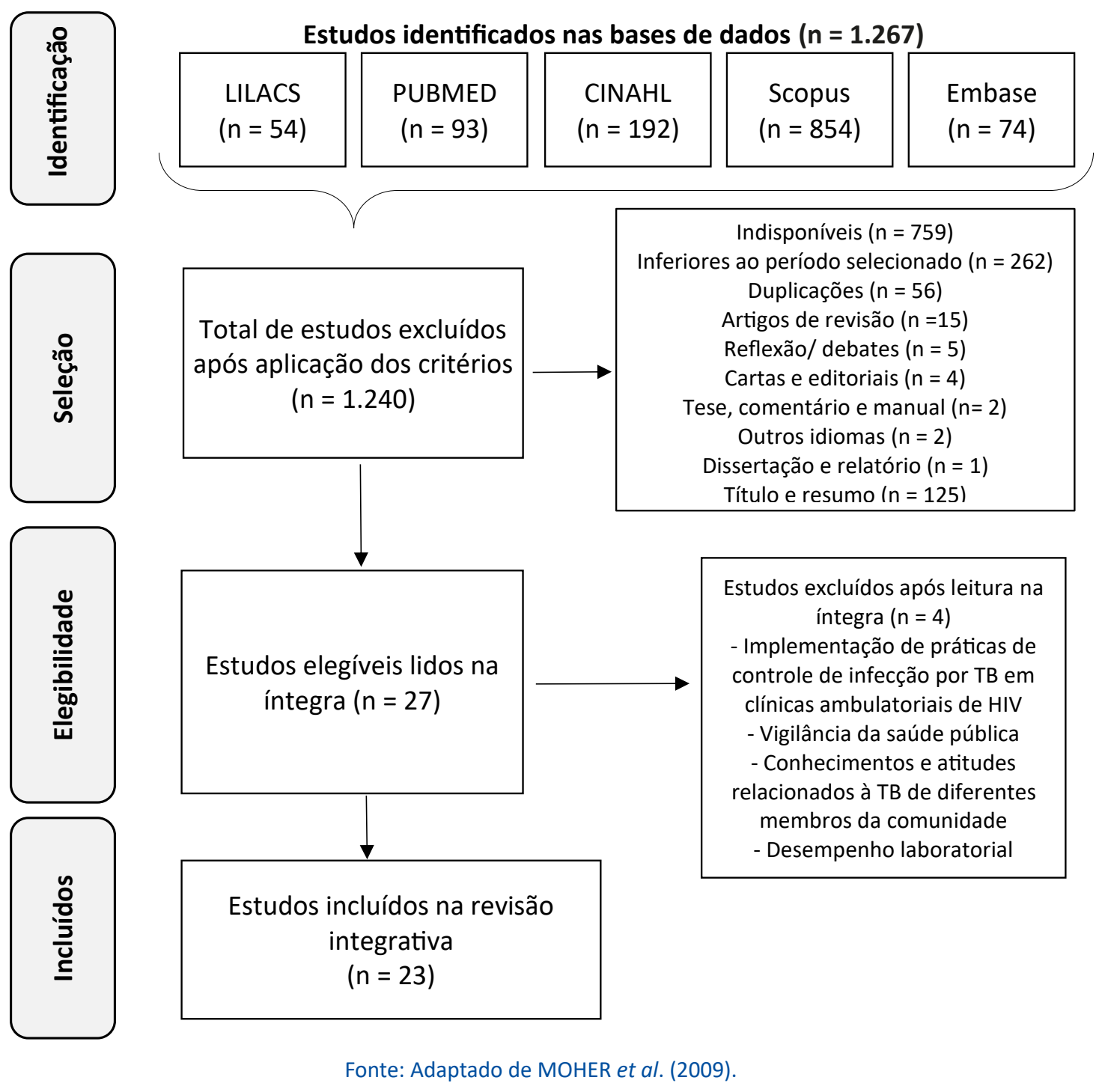

todos os casos mundialmente (WHO, 2019), a exemplo de Índia, Etiópia, Camboja, Nigéria, Libéria e Brasil (Tabela 1).

Quanto às características metodológicas dos estudos, identificou-se, sobretudo, pesquisas do tipo transversais $(30,4 \%)$, de avaliação em saúde $(26,1 \%)$ e estudos ecológicos (13\%). As demais envolveram estudos de casos múltiplos, de intervenção e coorte, variando entre abordagens qualitativas, quantitativas e métodos mistos (Tabela 1).

Os estudos apresentam aspectos relacionados ao desempenho dos PCTs quanto aos efeitos da instituição dos programas nos diferentes cenários estudados, levando em conta as populações de risco para o adoecimento pela TB, tais como as pessoas em situação de rua, Pessoas Vivendo com HIV (PVHIV) e a Po- pulação Privada de Liberdade (PPL), bem como o cenário da Atenção Primária à Saúde (APS). Além disso, o desempenho pôde ser avaliado a partir da satisfação dos usuários, da comunidade e dos profissionais de saúde, e de estratégias incorporadas aos programas para o controle da doença por meio do Directly Observed Treatment Short-Course (DOTS) (Tabela 1).

No cenário nacional foram identificadas algumas limitações a serem transpostas: realização do TDO, alocação de recursos para o PCT prisional, investimento nas equipes da APS, diagnóstico, assistência clínica e laboratorial. No cenário internacional detectou-se dificuldades estruturais e de processo, como o acesso aos serviços de saúde, busca ativa de casos e manejo da coinfecção TB/HIV, o que refletiu nos resultados obtidos pelos programas (Tabela 1). 
Tabela 1 - Características dos artigos incluídos nesta revisão integrativa da literatura, de acordo com os autores, ano, país e periódico de publicação, tipo de estudo e principais resultados, 2020

\begin{tabular}{|c|c|c|c|}
\hline Autores & $\begin{array}{l}\text { Ano, país e } \\
\text { periódico de } \\
\text { publicação }\end{array}$ & Tipo de estudo & Principais resultados \\
\hline $\begin{array}{c}\text { Candelária; } \\
\text { Llanes; Ranero }\end{array}$ & $\begin{array}{l}\text { 2014/ Cuba/ } \\
\text { Rev. Cuba. hig. } \\
\text { epidemiol }\end{array}$ & $\begin{array}{l}\text { Estudo } \\
\text { avaliativo } \\
\text { retrospectivo }\end{array}$ & $\begin{array}{l}\text { O desempenho do PNCT foi mal avaliado no ano de } 2006 \text {, uma vez que foram } \\
\text { observadas dificuldades de estrutura e deficiências de processo, o que refletiu nos } \\
\text { resultados obtidos pelo programa. }\end{array}$ \\
\hline Portela et al. & $\begin{array}{l}\text { 2014/ Brasil/ } \\
\text { Revista de Saúde } \\
\text { Pública }\end{array}$ & $\begin{array}{l}\text { Estudo } \\
\text { transversal }\end{array}$ & $\begin{array}{l}\text { Houve elevado nível de satisfação dos usuários quanto ao desempenho do PCT, } \\
\text { sobretudo com relação à provisão de medicamentos e respeito aos pacientes } \\
\text { pelos profissionais de saúde. Mostraram-se insatisfeitos pacientes mais jovens, } \\
\text { submetidos a tratamento autoadministrado e com nível superior. A satisfação } \\
\text { geral foi maior entre os pacientes sob DOTS. }\end{array}$ \\
\hline $\begin{array}{l}\text { Oliveira; Natal; } \\
\text { Camacho }\end{array}$ & $\begin{array}{l}\text { 2015a/ Brasil/ } \\
\text { Cadernos de Saúde } \\
\text { Pública }\end{array}$ & $\begin{array}{l}\text { Estudo } \\
\text { de casos } \\
\text { múltiplos/ } \\
\text { Qualitativo }\end{array}$ & $\begin{array}{l}\text { As ações do PCT não estiveram totalmente implantadas nas prisões com maior } \\
\text { PPL, altas taxas de incidência de TB e situação econômica melhor que a média do } \\
\text { país. }\end{array}$ \\
\hline $\begin{array}{l}\text { Oliveira; Natal; } \\
\text { Camacho }\end{array}$ & $\begin{array}{l}\text { 2015b/ Brasil/ } \\
\text { Revista de Saúde } \\
\text { Pública }\end{array}$ & $\begin{array}{l}\text { Estudo } \\
\text { de casos } \\
\text { múltiplos/ } \\
\text { Qualitativo }\end{array}$ & $\begin{array}{l}\text { Observou-se problemas no desempenho do PCT prisional, tais como baixa } \\
\text { proporções de recursos destinados às necessidades sanitárias, pouca participação } \\
\text { dos gerentes e profissionais de saúde nas decisões sobre aplicação dos recursos e } \\
\text { crescimento contínuo do número de PPL com TB. }\end{array}$ \\
\hline Hemmer et al. & $\begin{array}{l}\text { 2015/ Camarões/ } \\
\text { Asian Pacific Journal } \\
\text { of Tropical Disease }\end{array}$ & $\begin{array}{l}\text { Estudo } \\
\text { retrospectivo }\end{array}$ & $\begin{array}{l}\text { Com a integração do Programa Nacional de Controle da Aids com o PCT de } \\
\text { Camarões, houve melhora na detecção de casos de HIV e da prescrição de } \\
\text { cotrimoxazol entre pacientes coinfectados TB/HIV, diminuindo a mortalidade } \\
\text { nesse grupo vulnerável. }\end{array}$ \\
\hline $\begin{array}{l}\text { Mendonça; } \\
\text { Franco }\end{array}$ & $\begin{array}{l}\text { 2015/ Brasil/ } \\
\text { Epidemiol. serv. } \\
\text { saúde }\end{array}$ & $\begin{array}{l}\text { Estudo } \\
\text { ecológico }\end{array}$ & $\begin{array}{l}\text { Verificou-se a existência de diferentes níveis de risco de morbimortalidade e } \\
\text { índices de desempenho das ações de controle da TB nas Regiões de Saúde: seis } \\
\text { regiões de maior gravidade, nove intermediárias e seis de menor gravidade. }\end{array}$ \\
\hline $\begin{array}{c}\text { Dangisso; } \\
\text { Datiko; Lindtjørn }\end{array}$ & $\begin{array}{l}\text { 2015/ Etiópia/ } \\
\text { Global Health } \\
\text { Action }\end{array}$ & $\begin{array}{l}\text { Estudo } \\
\text { ecológico }\end{array}$ & $\begin{array}{l}\text { Foram utilizadas as taxas de notificação de casos de TB e os resultados do } \\
\text { tratamento para medir o desempenho do PCT no período de } 2003 \text { a } 2012 \text {. O acesso } \\
\text { aos serviços de saúde e intervenção ativa para deteç̧ão de casos influenciou no } \\
\text { aumento de notificação de casos e nos resultados do tratamento da doença. }\end{array}$ \\
\hline $\begin{array}{l}\text { Lavôr; Pinheiro; } \\
\text { Gonçalves }\end{array}$ & $\begin{array}{l}\text { 2016/ Brasil/ Rev. } \\
\text { esc. enferm. USP }\end{array}$ & $\begin{array}{l}\text { Estudo de } \\
\text { avaliação } \\
\text { de casos } \\
\text { múltiplos }\end{array}$ & $\begin{array}{l}\text { A implantação parcial }(74,7 \%) \text { da estratégia DOTS, na cidade de Manaus no ano de } \\
\text { 2012, refletiu na inconformidade do controle da TB, levando à baixa efetividade e } \\
\text { desempenho do PCT. }\end{array}$ \\
\hline Sunda & $\begin{array}{l}\text { 2017/ Camboja/ } \\
\text { Health Policy \& } \\
\text { Planning }\end{array}$ & $\begin{array}{l}\text { Estudo } \\
\text { transversal/ } \\
\text { Qualitativo }\end{array}$ & $\begin{array}{l}\text { No geral, as opiniões sobre os serviços de TB vinculados ao PCT foram positivas, } \\
\text { uma vez que houve melhora das percepções da doença e confiança nos serviços } \\
\text { de saúde. }\end{array}$ \\
\hline Oshi et al. & $\begin{array}{l}\text { 2017/ Nigéria/ } \\
\text { International } \\
\text { Journal of } \\
\text { Mycobacteriology }\end{array}$ & $\begin{array}{l}\text { Estudo de } \\
\text { intervenção }\end{array}$ & $\begin{array}{l}\text { A intervenção do estudo contribuiu para uma melhor detecção de casos de TB, } \\
\text { por meio da busca ativa em ambientes com recursos limitados e integração ao PCT } \\
\text { para melhora de seu desempenho. }\end{array}$ \\
\hline $\begin{array}{l}\text { Silva-Sobrinho } \\
\text { et al. }\end{array}$ & $\begin{array}{l}\text { 2017/ Brasil/ The } \\
\text { open nursing } \\
\text { journal }\end{array}$ & $\begin{array}{l}\text { Estudo } \\
\text { transversal/ } \\
\text { Quantitativo }\end{array}$ & $\begin{array}{l}\text { A avaliação do PCT de acordo com diferentes conjuntos de indicadores de estrutura } \\
\text { e processo mostra a necessidade de investir na equipe da APS e melhorar o } \\
\text { manejo clínico dos casos. }\end{array}$ \\
\hline Negandhi et al. & $\begin{array}{l}\text { 2017/ Índia/ Global } \\
\text { Health Action }\end{array}$ & $\begin{array}{l}\text { Estudo } \\
\text { qualitativo }\end{array}$ & $\begin{array}{l}\text { O PNCT revisado na Índia baseia-se na estratégia DOTS, por meio da mudança } \\
\text { de regime intermitente para o regime diário, desenvolvendo estratégias para } \\
\text { minimizar o estigma, incentivar a adesão, assegurar o monitoramento regular e } \\
\text { expandir a prestação de serviços de saúde de qualidade. }\end{array}$ \\
\hline Arakawa et al. & $\begin{array}{l}\text { 2017/ Brasil/ } \\
\text { Revista de Saúde } \\
\text { Pública }\end{array}$ & $\begin{array}{l}\text { Pesquisa de } \\
\text { avaliação em } \\
\text { saúde, com } \\
\text { delineamento } \\
\text { ecológico }\end{array}$ & $\begin{array}{l}\text { O grupo de pior desempenho apresentou as taxas mais elevadas de abandono } \\
\text { e as menores proporções de TDO, e esteve associado à baixa incidência de TB, } \\
\text { alta coinfecção TB/HIV, pequeno porte populacional, alta cobertura de ESF/PACS e } \\
\text { localização no interior. O grupo de melhor desempenho apresentou a maior taxa } \\
\text { de cura e a maior proporção de casos em TDO, enquanto o grupo de desempenho } \\
\text { regular mostrou resultados regulares de desfecho e de efetivação do TDO. }\end{array}$ \\
\hline $\begin{array}{l}\text { Mundra; } \\
\text { Deshmukh; } \\
\text { Dawale }\end{array}$ & $\begin{array}{l}2017 / \text { Índia/ Journal } \\
\text { of Epidemiology } \\
\text { and Global Health }\end{array}$ & $\begin{array}{l}\text { Estudo de } \\
\text { Coorte }\end{array}$ & $\begin{array}{l}\text { O PCT propôs o acompanhamento a longo prazo de pacientes a cada seis } \\
\text { meses para detectar os casos de recidivas precocemente, e que os membros da } \\
\text { família sejam os provedores do DOTS, a fim de reduzir a morbimortalidade e as } \\
\text { interrupções do tratamento, respectivamente, para melhorar o desempenho do } \\
\text { programa. }\end{array}$ \\
\hline
\end{tabular}




\begin{tabular}{|c|c|c|c|}
\hline $\begin{array}{l}\text { Queiroga; Sá; } \\
\text { Gazzinelli }\end{array}$ & $\begin{array}{c}\text { 2018/ Brasil/ Rev } \\
\text { Rene }\end{array}$ & $\begin{array}{l}\text { Pesquisa } \\
\text { avaliativa }\end{array}$ & $\begin{array}{l}\text { A análise de desempenho do PCT evidenciou a deficiência dos profissionais da } \\
\text { APS de Campina Grande no desenvolvimento de ações para o controle da TB na } \\
\text { população em situação de rua. }\end{array}$ \\
\hline Melese et al. & $\begin{array}{l}\text { 2018/ Etiópia/ } \\
\text { Journal of Clinical } \\
\text { Tuberculosis } \\
\text { and Other } \\
\text { Mycobacterial } \\
\text { Diseases }\end{array}$ & $\begin{array}{l}\text { Estudo de } \\
\text { intervenção }\end{array}$ & $\begin{array}{l}\text { A ferramenta de padrões de atendimento (SOC), desenvolvida na Etiópia, auxiliou } \\
\text { a identificar as lacunas do PCT, por meio de orientações quanto à qualidade do } \\
\text { atendimento que um paciente deve receber com base na OMS ou em indicadores } \\
\text { nacionais de desempenho. }\end{array}$ \\
\hline
\end{tabular}

\begin{tabular}{|c|c|c|c|}
\hline $\begin{array}{l}\text { Desta; Masango; } \\
\text { Nkosi }\end{array}$ & $\begin{array}{c}\text { 2018/ Libéria/ PLoS } \\
\text { ONE }\end{array}$ & $\begin{array}{l}\text { Estudo quanti } \\
\text { e qualitativo }\end{array}$ & $\begin{array}{l}\text { Os resultados do estudo apontaram que houve uma melhora geral no desempenho } \\
\text { do PCT na Libéria de } 2003 \text { a 2013, em relação à restauração dos serviços de TB, } \\
\text { resultados do tratamento e cobertura do DOTS, em contraposição aos serviços } \\
\text { laboratoriais e de recursos humanos. }\end{array}$ \\
\hline Motamedizadeh; & $\begin{array}{l}\text { 2018/ Irã/ Shiraz E } \\
\text { Medical Journal }\end{array}$ & $\begin{array}{l}\text { Estudo } \\
\text { transversal }\end{array}$ & $\begin{array}{l}\text { Estudo evidenciou que a TB pode ser eliminada por meio da instituição da } \\
\text { estratégia DOTS, programas educacionais e de medicamentos, além da execução } \\
\text { de políticas adequadas para o manejo da doença pelo PCT. }\end{array}$ \\
\hline Atekem et al. & $\begin{array}{l}\text { 2018/ Camarões/ } \\
\text { International } \\
\text { Journal of } \\
\text { Mycobacteriology }\end{array}$ & $\begin{array}{l}\text { Estudo } \\
\text { retrospectivo } \\
\text { transversal }\end{array}$ & $\begin{array}{l}\text { A taxa de sucesso do tratamento foi de } 83,4 \% \text {, não atingindo a meta da OMS, e } \\
\text { foi afetada pelo ano de início de tratamento, coinfecção TB/HIV, forma clínica e } \\
\text { serviço de tratamento, o que indicou a baixa eficácia do PCT. }\end{array}$ \\
\hline $\begin{array}{c}\text { Kim; Keshavjee; } \\
\text { Atun }\end{array}$ & $\begin{array}{l}\text { 2019/ } 183 \text { países/ } \\
\text { Journal of Global } \\
\text { Health }\end{array}$ & $\begin{array}{c}\text { Estudo } \\
\text { avaliativo }\end{array}$ & $\begin{array}{l}\text { Encontrou-se uma relação negativa geral entre os indicadores de saúde da } \\
\text { população, de modo que os países com cascatas e atendimento com baixo } \\
\text { desempenho tiveram piores resultados e cobertura dos serviços de saúde. }\end{array}$ \\
\hline Sharma et al. & $\begin{array}{l}2019 / \text { Índia/ } \\
\text { Indian journal } \\
\text { of community } \\
\text { medicine }\end{array}$ & $\begin{array}{l}\text { Estudo de } \\
\text { rastreamento } \\
\text { baseado na } \\
\text { comunidade }\end{array}$ & $\begin{array}{l}\text { O acompanhamento de pacientes curados pelo PCT detectou resultados adversos } \\
\text { altos. Os que sobreviveram e parentes dos casos de óbitos classificaram o } \\
\text { desempenho do programa como bom a muito bom. }\end{array}$ \\
\hline $\begin{array}{c}\text { Desta; Kessely; } \\
\text { Daboi }\end{array}$ & $\begin{array}{l}\text { 2019/ Libéria/ BMC } \\
\text { Public Health }\end{array}$ & $\begin{array}{l}\text { Estudo } \\
\text { transversal/ } \\
\text { Quali e } \\
\text { quantitativo }\end{array}$ & $\begin{array}{l}\text { Epidemias, como a do Ebola, impactaram negativamente o diagnóstico dos casos } \\
\text { de TB e o sucesso do tratamento entre } 2014 \text { e } 2015 \text {. A perda de seguimento caiu } \\
\text { para } 13 \% \text { em 2016, indicando uma melhora progressiva no desempenho do PCT. }\end{array}$ \\
\hline Bezerra et al. & 2019/ Brasil/ Pharm & $\begin{array}{l}\text { Estudo } \\
\text { transversal }\end{array}$ & $\begin{array}{l}\text { No geral, as perspectivas do paciente e do profissional foram desfavoráveis. } 0 \\
\text { primeiro pontuou aspectos relativos à acessibilidade organizacional de maneira } \\
\text { mais desfavorável, enquanto o segundo expressou menos opiniões positivas sobre } \\
\text { diagnóstico, assistência clínica e laboratorial }\end{array}$ \\
\hline
\end{tabular}

Legenda: ESF/Pacs (Estratégia Saúde da Família/ Programa de Agentes Comunitários de Saúde). OMS (Organização Mundial da Saúde). Fonte: Elaborada pelas autoras (2020).

\section{DISCUSSÃO}

Embora os PCTs tenham objetivos e diretrizes bem delimitados pelos governos de cada país, identificou-se elementos singulares quanto ao desempenho dos programas em cada cenário de estudo. Há, no entanto, uma dificuldade em definir se o desempenho seria a efetivação das ações específicas para o controle da TB, ou sobre o PCT em si, ou sobre os serviços que deveriam operacionalizá-lo.

Estudos abordam fragilidades para a instituição do PCT nos diferentes cenários estudados, os quais revelam que o desempenho insatisfatório dos programas influencia na qualidade da assistência à saúde e nos modelos assistenciais que vêm sendo praticados na realidade dos serviços de saúde (ARAKAWA et al., 2017; CANDELÁRIA; LLANES; RANERO, 2014; MENDONÇA; FRANCO, 2015).
A análise dos elementos de estrutura, processo e resultado de Donabedian permitiu identificar deficiências à luz da literatura, em que as dificuldades de estrutura estiveram relacionadas à baixa cobertura de saúde da população, educação permanente às equipes de saúde, falta de recursos financeiros e laboratórios adequados e, as de processo, relacionadas à pouca atividade de educação em saúde aos usuários, desarticulação dos serviços de saúde, atitude dos profissionais de saúde, bem como ao tempo para o diagnóstico e início do tratamento (CANDELÁRIA; LLANES; RANERO, 2014; SILVA-SOBRINHO et al., 2017; DESTA; MASANGO; NKOSI, 2018), o que reflete nos resultados obtidos pelos programas.

Nesse sentido, questiona-se a capacidade estrutural, gerencial e organizacional para a operacionalização do PCT para o alcance de metas quanto à diminuição de casos novos, abandono, óbito e aumento das taxas de cura (ARAKAWA et al., 2017), uma vez que 
a fragmentação das práticas, o despreparo para lidar com os doentes de TB, bem como para executar ações de diagnóstico, assistência e prevenção, ainda se apresentam pertinentes (SILVA-SOBRINHO et al., 2017).

Levando em conta que os serviços de saúde não são igualmente distribuídos e acessíveis a todos os usuários da comunidade, um estudo realizado no sul da Etiópia (2015) afirma que o maior acesso aos serviços de controle da TB e sua relação com o aumento de casos diagnosticados e notificados auxiliam nos resultados do tratamento, redução dos casos infecciosos e, consequentemente, na melhora do desempenho dos programas.

As ações específicas do programa devem objetivar o controle da doença, por meio do alcance das metas estabelecidas pela OMS, preconizadas até 2015: coeficiente de incidência da doença para <10 casos/ 100 mil hab. e <1 óbito/ 100 mil hab., assim como taxas de até $5 \%$ para o abandono do tratamento e, no mínimo, 85\% para a cura (WHO, 2015). Estudos ressaltam que, a taxa de sucesso do tratamento da TB é considerada um dos melhores indicadores de resultado dos PCT, o que auxilia na avaliação de seus desempenhos (ATEKEM et al., 2018; DESTA; MASANGO; NKOSI, 2018; DESTA; KESSELY; DABOI, 2019).

Desfechos desfavoráveis para o tratamento, entretanto, tais como o abandono, óbito, recidiva e retratamento são obstáculos para o alcance dessas metas, sobretudo quando relacionados a baixos desempenhos dos PCTs quanto à organização de recursos humanos, sobrecarga de trabalho, qualidade do registro de dados, cobertura do TDO, menos interação, aconselhamento, suporte e troca de informações entre doente e profissionais (MUNDRA; DESHMUKH; DAWALE, 2017; ATEKEM et al., 2018).

Isto indica que os programas necessitam inserir estratégias eficazes de rastreamento e abordagens de tratamento centrado nos doentes de TB (DESTA; MASANGO; NKOSI, 2018), bem como revisões nos programas e treinamento para os profissionais de saúde (MOTAMEDIZADEH; ALIMOHAMMADZADEH; HOSSEINI, 2018), visando a atender às exigências nacionais e internacionais e as metas globais da OMS perante o diagnóstico e tratamento de doenças endêmicas, tal como a TB (DESTA; KESSELY; DABOI, 2019).

Avaliar a satisfação dos usuários em relação ao cuidado recebido e identificar barreiras para a adesão ao tratamento constitui-se um desafio. Assim, estudos esclarecem que aspectos estruturais - limpeza, conforto, disponibilidade de exames na unidade e aumento no número de médicos - e organizacionais - diminuição do tempo de espera para consulta mar- cada, assistência clínica e laboratorial - devem ser considerados para o aprimoramento do PCT visando ao aumento de sua efetividade perante os usuários e profissionais de saúde (PORTELA et al., 2014; BEZERRA et al., 2019).

Embora as opiniões sobre os serviços de TB sejam positivas em alguns estudos, a existência de dicotomias nas experiências e comportamento dos usuários e comunidade em relação à procura de cuidados são indicativos de barreiras de acesso ao diagnóstico e tratamento precoce, e má qualidade percebida, o que pode ser trabalhado com o fortalecimento de serviços horizontais de saúde (SUNDARAM et al., 2017; SHARMA et al., 2019).

Considerando algumas populações vulneráveis com maior risco de adoecimento para a TB - as pessoas em situação de rua (56x), PVHIV (28x) e PPL (28x) (BRASIL, 2018a), estudos destacam a necessidade de investimento no PCT para ações específicas e articulações intersetoriais com o objetivo de conter esta epidemia global (OLIVEIRA; NATAL; CAMACHO, 2015a).

Mesmo a população em situação de rua sendo considerada prioritária para o PNCT devido à maior vulnerabilidade social e suscetibilidade a infecções, resultados encontrados em Campina Grande (PB) mostram que a análise de desempenho do programa evidenciou deficiências dos profissionais da APS no desenvolvimento de ações para o controle da doença, por não considerarem as especificidades dessa população, que se somam a fragilidades quanto à capacitação dos profissionais, deficiente acesso aos manuais, ausência de articulação e apoio das equipes de consultório de rua (QUEIROGA; SÁ; GAZZINELLI, 2018).

Desempenhos insatisfatórios associados com uma alta coinfecção TB/HIV apontam para a necessidade de maior vigilância e aperfeiçoamento no manejo dos que são mais vulneráveis, com maior risco clínico, de efeitos adversos e desfechos como abandono e óbito (ARAKAWA et al., 2017).

Para diminuir a carga do HIV em pacientes com TB, a OMS recomendou cinco atividades: testagem e aconselhamento para HIV; introdução de métodos de prevenção do HIV; introdução da terapia preventiva com cotrimoxazol para as PVHIV; atendimento e apoio ao HIV/aids e introdução da Terapia Antirretroviral (TARV), as quais foram introduzidas e monitoradas em um estudo realizado em Camarões, revelando a melhora efetiva da detecção dos casos de HIV e tratamento profilático para a coinfecção, reduzindo significativamente a mortalidade nesse grupo de doentes (HEMMER et al., 2015). 
Estudos realizados em presídios brasileiros afirmam que, entre as PPL, mesmo preconizado a instituição do PCT no sistema prisional visando a garantir a detecção e diagnóstico precoces, isolamento, acompanhamento dos casos e planejamento da alta, ainda se observam cenários de altas taxas de incidência de $T B$, os quais demandam maior atenção quanto às ações de busca ativa no ingresso dentro do presídio, TDO, educação em saúde e comunicação entre profissionais de segurança, de saúde, unidades prisionais e sistema de saúde, visando a aumentar as taxas de cura e reduzir a transmissão da doença intra e extramuros (OLIVEIRA; NATAL; CAMACHO, 2015a, b).

Fatores como superlotação, condições ambientais precárias e carência de recursos, no entanto, constituem barreiras para a efetivação destas ações, que deveriam ser planejadas, gerenciadas e financiadas pelos Ministérios da Justiça e da Saúde, e Secretarias Estaduais de Administração Penitenciária e de Saúde, visando ao controle da doença nos ambientes prisionais (OLIVEIRA; NATAL; CAMACHO, 2015b).

A predominância do tratamento autoadministrado em comparação ao TDO, um dos pilares da estratégia DOTS (PORTELA et al., 2014), vai contra as recomendações para o tratamento da TB pela OMS. A introdução parcial da estratégia nos serviços de saúde e a falta de integração do PCT com a ESF na busca e acompanhamento dos casos refletem-se na inconformidade do controle da doença, levando à baixa efetividade dos programas (LAVÔR; PINHEIRO; GONÇALVES, 2016).

Mesmo que a literatura destaque que os doentes de TB submetidos ao TDO conseguem maior adesão ao tratamento (PORTELA et al., 2014), melhor desfecho como resultado do tratamento (ARAKAWA et al., 2017), enfrentamento da resistência aos medicamentos e recorrência da TB (MOTAMEDIZADEH; ALIMOHAMMADZADEH; HOSSEINI, 2018), ainda se observa a priorização nas ações programáticas, em detrimento da autonomia técnica, política e financeira, com auxílio transporte e/ou alimentação (LAVÔR; PINHEIRO; GONÇALVES, 2016).

Por outro lado, Negandhi et al. (2017) afirmam que na Índia, pelo fato do PCT ser baseado na estratégia DOTS, houve uma notável melhoria nos resultados do programa com um rápido progresso em todo o país, uma vez que o programa é bem apoiado por comprometimento político proativo, financiamento e monitoramento regular, com medicamentos de alta qualidade oferecidos durante todo o tratamento.
A busca ativa de casos na comunidade desempenha um importante papel no aumento das notificações de casos e nos resultados do tratamento (DANGISSO; DATIKO; LINDTJ ØRN, 2015). Estudo realizado na Nigéria (2017) aponta que a busca ativa de casos na comunidade e triagem sistemática de casos de TB, especialmente em ambientes de alta prevalência e com recursos limitados, são necessárias para melhorar a deteç̧ão e tratamento dos casos, em oposição à busca passiva, além de gerarem evidências para a integração, ou não, ao PNCT como estratégia política para o controle da doença em países com características socioeconômicas e epidemiológicas semelhantes.

Considerando que dados de boa qualidade são necessários para monitorar o desempenho dos PCT, um estudo realizado na Etiópia (2018) desenvolveu uma ferramenta de orientação baseada em evidências e supervisão para gerentes identificarem as lacunas do programa e priorizar as unidades de saúde que receberão recursos com base em seu desempenho, o que permitiu melhorar a localização dos casos de TB, resultados do tratamento, gerenciamento de medicamentos, qualidade laboratorial, controle de infecção e desempenho do programa em si.

Kim, Keshavjee e Atun (2019), em um estudo realizado em 183 países, afirmam que a ferramenta baseada em cascatas de atendimento à TB, baseada em quatro etapas - incidência, diagnóstico, tratamento iniciado e concluído - é eficaz para o manejo bem-sucedido da doença e útil para avaliar o desempenho dos PCTs e sistemas de saúde. Assim sendo, estratégias para o controle da doença utilizadas nos diferentes cenários estudados, para a população geral ou vulnerável para o adoecimento da TB, podem ser aplicadas pelos PCTs para melhora de seu desempenho e cumprimento das metas globais.

\section{CONSIDERAÇÕES FINAIS}

Esta revisão permitiu identificar elementos singulares quanto ao desempenho dos PCTs em cada cenário de estudo, mesmo havendo uma dificuldade em definir, de um ponto específico, o conceito de desempenho. Possuiu como limitação a inclusão de artigos somente em livre acesso, o que pode ter ocasionado a exclusão de alguns estudos que abordassem a temática.

Por fim, aponta para a importância da construção de debates sobre os desafios que ainda permeiam o sistema de saúde e a sociedade, visando à instauração de ações integradas e intersetoriais na efetivação do combate e controle da doença, levando em conta 
os determinantes da TB que requerem uma abordagem interprofissional e centrada nos doentes, família e comunidade.

\section{REFERÊNCIAS}

ARAKAWA, T et al. Tuberculosis control program in the municipal context: performance Evaluation. Rev. Saúde Pública, v. 51, n. 0, p. 23, 2017.

ARAKAWA, T. et al. Avaliação de desempenho de Programas de Controle de Tuberculose no contexto brasileiro e espanhol: uma revisão integrativa da literatura. Ciência \& Saúde Coletiva, v. 20, n. 12, p. 3.877-3.889, 2015.

ATEKEM, K. A. et al. Evaluation of the tuberculosis control program in South West Cameroon: Factors affecting treatment outcomes. International Journal of Mycobacteriology, v. 7, n. 2, p. 137-142, 2018.

BEZERRA S. S. et al. The perspectives of patients and health professionals regarding the tuberculosis control programme in Recife, Brazil: A contribution to Evaluation. Pharm., v. 7, n. 2, 2019.

BRASIL. Ministério da Saúde. Secretaria de Vigilância em Saúde. Departamento de Vigilância das Doenças Transmissíveis. Manual de Recomendações para o Controle da tuberculose no Brasil. Brasília, 2018a.

BRASIL. Ministério da Saúde. Secretaria de Vigilância em Saúde. Implantação do Plano Nacional pelo Fim da Tuberculose como Problema de Saúde Pública no Brasil: primeiros passos rumo ao alcance das metas. Brasília, v. 49, n. 11, 2018b.

CANDELARIA, S. R.; LLANES, L. G.; RANERO, V. M. Evaluación del Programa Nacional de Control de la Tuberculosis en Bauta. Revista Cubana de Higiene y Epidemiología, v. 52, n. 1, p. 98-105, 2014.

DANGISSO, M. H.; DATIKO, D. G.; LINDTJøRN, B. Accessibility to tuberculosis control services and tuberculosis programme performance in southern Ethiopia. Global Health Action, v. 8, p. 29.443, 2015.

DESTA, K. T.; KESSELY, D. B.; DABOI, J. G. Evaluation of the performance of the National Tuberculosis Program of Liberia during the 2014-2015 Ebola outbreak. BMC Public Health, v. 19, n. 1, 2019.

DESTA, K. T.; MASANGO, T. E.; NKOSI, Z. Z. Performance of the national tuberculosis control program in the post conflict Liberia. PLOS ONE, v. 13, n. 6, p. e0199474, 2018.

HEMMER, C. J. et al. Integration of HIV services into the National Tuberculosis Program of Cameroon: The experience of the Littoral Province. Asian Pacific Journal of Tropical Disease, v. 5, n. 7, p. 525-528, 2015.

HOPIA, H.; LATVALA, E.; LIIMATAINEN, L. Reviewing the methodology of an integrative review. Scand J Caring Sci, v. 30, n. 4, p. 662-669, 2016.

KIM, J.; KESHAVJEE, S.; ATUN, R. Health systems performance in managing tuberculosis: Analysis of tuberculosis care cascades among high-burden and non-highburden countries. Journal of Global Health, v. 9, n. 1, 2019.
LAVÔR, D. C. B. S.; PINHEIRO, J. S.; GONÇALVES, M. J. F. Evaluation of the implementation of the directly observed treatment strategy for tuberculosis in a large city. Rev. Esc. Enferm. USP, v. 50, n. 2, p. 245-252, 2016.

MELESE, M. et al. Use of indicators of standards of care to improve tuberculosis program management in Ethiopia. Journal of Clinical Tuberculosis and Other Mycobacterial Diseases, v. 10, n. 0, p. 17-23, 2018.

MENDONÇA, S. A.; FRANCO, S. C. Avaliação do risco epidemiológico e o desempenho dos programas de controle de tuberculose nas Regiões de Saúde do estado de Santa Catarina, 2003 a 2010. Epidemiol. Serv. Saúde, v. 24, n. 1, p. 59-70, 2015.

MOHER, D. et al. Preferred Reporting Items for Systematic Reviews and MetaAnalyses: The PRISMA Statement. PLOS Med., v. 6, n. 7, p. e1000097, 2009.

MOTAMEDIZADEH, F.; ALIMOHAMMADZADEH, K.; HOSSEINI, S. M. Monitoring and evaluation of program performance and management control system of tuberculosis: A ten-year cross-sectional study in sistan and baluchestan province, Iran. Shiraz e Medical Journal, v. 19, n. 9, p. e60497, 2018.

MUNDRA, A.; DESHMUKH, P. R.; DAWALE, A. Magnitude and determinants of adverse treatment outcomes among tuberculosis patients registered under Revised National Tuberculosis Control Program in a Tuberculosis Unit, Wardha, Central India: A record-based cohort study. Journal of Epidemiology and Global Health, v. 7, n. 2, p. 111-118, 2017.

NEGANDHI, H. et al. Rapid assessment of facilitators and barriers related to the acceptance, challenges and community perception of daily regimen for treating tuberculosis in India. Global Health Action, v. 10, n. 1, 2017.

OLIVEIRA, L. G. D.; NATAL, S.; CAMACHO, L. A. B. Analysis of the implementation of the Tuberculosis Control Program in Brazilian prisons. Cad. Saúde Pública, v. 31, n. 3, p. 543-554, 2015a.

OLIVEIRA, L. G. D.; NATAL, S.; CAMACHO, L. A. B. Implementation contexts of a tuberculosis control program in Brazilian prisons. Revista de Saúde Publica, v. 49, n. 66, 2015b.

$\mathrm{OSHI}$, D. et al. An evaluation of innovative community-based approaches and systematic tuberculosis screening to improve tuberculosis case detection in Ebonyi State, Nigeria. International Journal of Mycobacteriology, v. 6, n. 3, p. 246-252, 2017.

OUZZANI, M. et al. Rayyan - a web and mobile app for systematic reviews. Syst Revi., 2016, v. 5, n. 1, p. 1-10. Disponivel em: https://www.ncbi.nlm.nih.gov/pmc/articles/ PMC5139140/pdf/13643_2016_Article_384.pdf. Acesso em: 18 fev. 2020.

PAIM, J. S. A constituição cidadã e os 25 anos do Sistema Único de Saúde (SUS). Cad. Saúde Pública, v. 29, n. 10, p. 1.927-1.936, 2013.

PAULA, C. C.; PADOIN, S. M. M.; GALVÃO, C. M. Revisão integrativa como ferramenta para tomada de decisão na prática de saúde. In: LACERDA, M. R.; COSTENARO, R. G. S. (org.). Metodologias da pesquisa para a enfermagem e saúde: da teoria à prática. Porto Alegre: Moriá, 2016. p. 51-76. 
PORTELA, M. C. et al. Tuberculosis control program and patient satisfaction. Revista de Saúde Pública, Rio de Janeiro, v. 48, n. 3, p. 497-507, 2014.

QUEIROGA, R. P. F.; SÁ, L. D.; GAZZINELLI, A. A tuberculose na população em situação de rua: desempenho de profissionais da atenção primária. Rev. Rene (On-line), v. 19, n. 0, p. e32463-e32463, 2018.

SHARMA, R. et al. An Outcome-Based Follow-up Study of Cured Category I Pulmonary Tuberculosis Adult Cases from Various Tuberculosis Units under Revised National Tuberculosis Control Program from a Western Indian City. Indian $J$ Community Med., v. 44, n. 1, p. 48-52, 2019.

SILVA-SOBRINHO, R. A. et al. Assessment of Primary Health Care in the Treatment of Tuberculosis in a Brazilian Locality of the International Triple Frontier. Open Nurs J., v. 22, n. 11, p. 124-134, 2017.

SUNDARAM, N. et al. A strong TB programme embedded in a developing primary healthcare system is a lose-lose situation: insights from patient and community perspectives in Cambodia. Health Policy \& Planning, v. 32, p. 32-42, 2017.

URSI, E. S. Prevenção de lesões de pele no perioperatório: revisão integrativa da literatura. 2005. 130 f. Dissertação (Mestrado em Enfermagem) - Escola de Enfermagem, Universidade de São Paulo, Ribeirão Preto, 2005.

VIACAVA, F. et al. Avaliação de desempenho de sistemas de saúde: um modelo de análise. Cien. Saúde Colet, v. 17, n. 4, p. 921-934, 2012.

WHO. World Health Organization. Global Tuberculosis Report 2019. Geneva: WHO, 2019.

WHO. World Health Organization End TB Strategy. Geneva: WHO, 2015. Disponível em: http://www.who.int/tb/ post2015_strategy/en/. Acesso em: 2 fev. 2020. 